
\title{
25 Research Soure \\ Cardiac biomarkers for early detection of cardiac involvement in children with Kawasaki disease: a cross-sectional study
}

\section{Akbar Molaei}

Tabriz University of Medical Sciences

Azizollah Khomahani

Tabriz University of Medical Sciences

Shamsi Ghaffari

Tabriz University of Medical Sciences

Mahnaz Sadeghi-Shabestari

Tabriz University of Medical Sciences

Seyyed-Reza Sadat-Ebrahimi ( $\sim$ Sadatr@tbzmed.ac.ir )

Tabriz University of Medical Sciences https://orcid.org/0000-0002-6697-4372

Research article

Keywords: Kawasaki disease, Cardiac troponin I, N-terminal pro-brain natriuretic peptide, Coronary artery aneurysm

Posted Date: August 4th, 2019

DOI: https://doi.org/10.21203/rs.2.11860/v1

License: (c) (1) This work is licensed under a Creative Commons Attribution 4.0 International License. Read Full License

Version of Record: A version of this preprint was published on November 12th, 2019. See the published version at https://dx.doi.org/10.22038/ijp.2019.43387.3617. 


\section{Abstract}

Background Kawasaki disease (KD), which is the second most prevalent vasculitis disease in children after IgA vasculitis, can cause serious cardiovascular complications. Early detection of cardiac involvement in $\mathrm{KD}$ is an essential part in managing and preventing the cardiac sequels. Although some cardiac biomarkers such as cardiac troponin I (cTnl) and N-terminal pro-brain natriuretic peptide (NTproBNP) have recently been suggested for early diagnosis of cardiac involvement in patients with KD, their applicability is still unclear. Thus, this study aimed to compare the levels of cTnl and NT-proBNP in KD child patients with or without cardiac involvement. Methods In this cross sectional study, 32 children with KD who were admitted to the children teaching hospital of Tabriz University of Medical Sciences between April 2015 and April 2018, were consecutively included in the study. For all involved children, the coronary artery involvement defined as coronary artery aneurysm or perivascular brightness of coronary arteries was examined by transthoracic echocardiography, and their serum levels of NT-proBNP and cTnl were measured. Results Of 32 enrolled patients, 4 (9.4\%) had cardiac involvement including 3 patients with perivascular brightness of coronary arteries and 1 patient with small aneurysm of the coronary arteries. In all study patients, the cTnl levels were lower than 0.35 and the NT-proBNP assessments revealed an average of $678.5 \mathrm{pg} / \mathrm{ml}$. Children with cardiac involvement had significantly higher NTproBNP ( $p$-value, 0.001 ). ROC analysis for power of the NT-proBNP in predicting the cardiac involvement, revealed an excellent power for NT-proBNP $(A U C=1.000, p=0.001)$. Both sensitivity and specificity of NTproBNP at the optimum cut-off point of $1354 \mathrm{pg} / \mathrm{ml}$ were 100 percent. Conclusion Unlike the cTnl, the NTproBNP can serve as an excellent objective test for early detection of cardiac involvement. Therefore, in KD-patients with high levels of NT-proBNP additional therapy and closer follow up should be considered.

\section{Background}

Although Kawasaki Disease (KD) is the second most prevalent vasculitis disease in children after IgA vasculitis (IgAV, Henoch-Schönlein purpura), it is known as the main cause of acquired cardiac disease in pediatrics (1-3). KD is typically a self-limiting diseases and the majority of patients show a complete recovery after the acute phase, but in some cases they disclose serious cardiovascular involvement both in acute and chronic stages (4).

$\mathrm{KD}$ is more common among the Asian children under 5 years of age (1), and approximately $15-25$ percent of children with untreated KD will develop coronary aneurysms. Although the coronary aneurysms in those children could be reduced by intravenous immunoglobulin (IVIG), five percent of those treated still develop coronary aneurysms (4). Moreover, histopathological findings has reported diffuse myocardial inflammation $(5,6)$. Also, some studies have reported diastolic dysfunction in $\operatorname{KD}$ patients $(7,8)$.

It has been postulated that early diagnosis and management of KD can prevent the further serious complications (9). According to the American Heart Association' guidelines, the diagnosis of classic KD is based on clinical manifestations including a prolonged fever that lasts more than five or four days along with four of five of the principal characteristics including the changes in extremities (erythema of palms 
and soles or edema of hands and feet in acute phase; periungual peeling of fingers and toes in sub-acute phase), polymorphous exanthema, bilateral bulbar conjunctival injection without exudate, erythema and cracking of lips, strawberry tongue, and/or erythema of oral and pharyngeal mucosa, unilateral cervical lymphadenopathy with diameter greater than $1.5 \mathrm{~cm}(3,9)$. In the atypical or incomplete form of KD, patients have prolonged fever with less than four of five principal clinical signs (9).

Furthermore, some laboratory findings such as elevated erythrocyte sedimentation rate, C-reactive protein level, hyponatremia and hypoalbuminemia could be helpful in diagnosis of suspected KD patients. Recently, several biomarkers have been suggested for diagnosis of KD including serum T-helper 1 and Thelper 2 cytokines (interleukin 6 and 20, tumor necrosis factor- $\alpha$ and interferon- $\gamma$ ) and serum N-terminal pro-brain natriuretic peptide (NT-proBNP) (10-12).

Early detection of cardiac involvement of KD is also important in managing and preventing the cardiac sequels in KD patients (13). Transthoracic echocardiography (TTE) is a diagnostic imaging modality of choice for examining the coronary aneurysms; however, more objective diagnostic tools are required for early detection of cardiac involvement in KD patients (3). It is more than a decade that elevated cardiac troponin I (cTnl) has been considered as a reliable marker of cardiac disorders (14), since it is the most sensitive and specific laboratory test for diagnosing the myocardial infarction (14). It has been demonstrated that NT-proBNP is a proper marker of congestive heart failure and it is elevated in acute myocardial injuries $(15,16)$. Also, it has been indicated that NT-proBNP has a high diagnostic value for cardiac involvement in some diseases such as amyloidosis and Marfan syndrome (17).

Both cTnl and NT-proBNP have been suggested by some studies as the predictors of cardiac involvement in KD. Although, Kim et al demonstrated that cTnl level significantly increases in KD patients especially in those with symptomatic myocarditis (18), Checchia et al did not show any the significant findings through the cTnl elevation in KD patients (19). Also, Kaneko et al. revealed that NT-proBNP level significantly increases in patients who have developed coronary artery lesion; Their findings were lately confirmed by the study of Adjagba et al. and Jung et al. (20-22). In contrast, a study by Iwashima et al did not present any significant differences in levels of NT-proBNP between KD patients with and without coronary artery lesions, however, those patients who developed mitral regurgitation showed significant higher levels of NT-proBNP (21). To date, none of cTnl and NT-proBNP biomarkers has been adequately evaluated for their clinical applicability in early diagnosis of cardiac involvement in KD patients. Thus, this study aimed to compare the levels of cTnI and NT-proBNP in KD patients with or without cardiac involvement and the association of them with the severity of cardiac disorders.

\section{Methods}

In this cross-sectional study, children with KD who were admitted to the children teaching hospital of Tabriz University of Medical Sciences between April 2015 and April 2018, were consecutively included in the study. Diagnosis of KD was made based on the American Heart Association' criteria for classic KD in children (3). The patients with a pervious history of congenital heart disease, uncorrected structural heart 
abnormality, dilated or hypertrophic cardiomyopathies, a previous episode of KD or carditis, and the ones who were recently treated with IVIG, and those undergoing or had undergone chemotherapy involving cardio-toxic drugs were excluded from the study. Also, the patients with a final diagnosis of incomplete $\mathrm{KD}$ due to its ambiguity were excluded as well.

In included KD patients, the coronary artery involvement was evaluated by a pediatric cardiologist using a TTE (Vivid- 3, General Electric Co., USA), and coronary arteries were measured and the Z-values were calculated with a similar method described by Miura et al. (23). Cardiac involvement was defined as the intra-luminal diameter of z-score of $\geq 2.5$ (coronary artery aneurysm) or perivascular brightness of coronary arteries (24). Also, we classified the coronary artery abnormalities as small if the $z$-score was $\geq$ 2.5 to $<5$, large if the $z$-score was $\geq 5$ to $<10$, and giant if the $z$-score was $\geq 10(25)$.

Demographic data and KD information were recorded for each child. The onset of KD was defined as the onset of fever in the children. Blood specimens ( $3 \mathrm{~mL}$ from each patient) were obtained at time of admission before any treatment intervention. Serum levels of NT-proBNP were analyzed using an electrochemiluminescence immunoassay with ECLusys 2010 analyzer (Roche Diagnostics, Indianapolis, IN); the manufacturer recommended the $125 \mathrm{pg} / \mathrm{ml}$ as cut-off point for NT-proBNP level. CTnl levels were assessed using the Stratus fluorometric enzyme immunoassay (Dade Pharmaceuticals, China). The manufacturer recommended $1.5 \mathrm{ng} / \mathrm{ml}$ as the cut-off point for cTnl level in diagnosing the myocardial disorder. The lower bond of detection by the applied immunoassay was $0.35 \mathrm{ng} / \mathrm{ml}$.

Data were analyzed using SPSS version 22. Normal numerical data were reported as mean \pm standard deviation and un-normal ones were reported as median (minimum-maximum). Categorical data were reported as frequency (percentage). Statistical comparisons between the two groups (with or without cardiac involvement) were performed by a two-tailed Student's t-test for numerical data and a Fisher's exact test for categorical data. The power of predicting cardiac involvement was evaluated based on the serum levels of NT-proBNP using the receiver operating characteristic curve (ROC). Also, their areas under the curve (AUCs) and specificity and sensitivity were calculated. A P-value of less than 0.05 was considered as significant.

\section{Results}

Among 54,124 patients admitted during the study period, 41 patients were diagnosed as KD. However, 9 patients were excluded due to the following reasons: 5 patients had a pervious history of congenital heart disease, 4 patients had a previous episode of carditis. Of 32 included patients, 8 (25\%) ones were female and $24(75 \%)$ ones were male, with a mean age of $3.38 \pm 1.96$ years old. None of the included patients had a significant medical history except for one patient that had cystic fibrosis. 4 (9.4\%) patients had cardiac involvement including 3 patients with perivascular brightness of coronary arteries and 1 patient with small aneurysm of the coronary arteries. One of the patients with perivascular brightness of coronary had also developed tricuspid valve regurgitation (TR). 
In all study patients, the cTnl levels were lower than 0.35 and the NT-proBNP levels were over than 125 $\mathrm{pg} / \mathrm{ml}$ exceeding normal ranges. The NT-proBNP measurements revealed a median of 678.5 (220-5735) $\mathrm{pg} / \mathrm{ml}$. Also, the highest level of NT-proBNP belonged to the patient who had perivascular brightness of coronary arteries (5735) beside. The other two patients with perivascular brightness of coronary arteries had NT-proBNP levels of 2427 and $5276 \mathrm{pg} / \mathrm{ml}$. The level of NT-proBNP in the patient with small aneurysm of coronary arteries was 1427.

The means of age in the patients with and without cardiac involvement were $2 \pm 2$ and $3 \pm 2$, respectively, which had no statistical difference $(\mathrm{p}=0.343$, Table 1). Of those without cardiac involvement, $21(72.4 \%)$ children were male and 8 (27.6\%) were female; however, all children with cardiac involvement were male $(p=0.001$, Table 1). Patients with cardiac involvement had significantly higher levels of NT-proBNP $(p=0.001$; Figure 1). ROC analysis for power of the NT-proBNP in predicting the cardiac involvement in children with KD, revealed an excellent power for NT-proBNP ( $A U C=1.000, p=0.001$, Figure 2). Both sensitivity and specificity of NT-proBNP at the optimum cut-off point of $1354 \mathrm{pg} / \mathrm{ml}$ were 100 percent.

\section{Discussion}

The current study investigated the applicability of NT-proBNP and cTnl levels in diagnosing the cardiac involvement in children with KD. Our results demonstrated that NT-proBNP was elevated in KD patients particularly in those with cardiac involvement including perivascular brightness of coronary arteries or coronary artery aneurysm; however, cTnl was in the normal range in all patients regardless of cardiac involvement. NT-proBNP showed an excellent power in predicting the presence of cardiac involvement in KD patients. There were no significant differences difference between the KD patients with and without cardiac involvement in age and the average days between onset of KD and referring to hospital. The majority of patients without cardiac involvement and all patients with cardiac involvement were males. It has been indicated that males are in a higher risk of developing coronary artery aneurysm than females (26).

Our results were in agreement with the pervious findings in value of NT-proBNP in predicting the presence of cardiac involvement (20-22). However, they had limited the definition of cardiac involvement to the development of coronary artery aneurysm but we also assessed the presence of perivascular brightness of coronary arteries as a preceding factor of developing of coronary artery aneurysm or subsequent coronary ectasia $(27,28)$. Though detecting this abnormality in echocardiography is qualitative and its evaluation depends on the experience of individual echocardiographers. Therefore, an objective test like serum NT-proBNP could be a useful substitute in this regard.

Although Iwashima et al. found no significant difference in levels of the NT-proBNP between those patients with and without coronary artery lesions, they reported that those patients with valvular dysfunction had significantly higher levels of NT-proBNP (21). Also, in our study, the highest level of NTproBNP was belonged to a patient with TR. It is postulated that TR in KD patients could be a great predictor in their ICU admission (29). Therefore, we expected NT-proBNP levels to be higher in those with 
more severe cardiac damage. However, considering that the NT-proBNP levels in other patient with sole perivascular brightness of coronary arteries (without TR) were more than the patient with small aneurysm of coronary arteries, we could not conclude a meaningful association between NT-proBNP levels and the severity of cardiac involvement in our study. Although, if we could enroll more KD patients with cardiac involvement, declaring that association could be more accurate.

ProBNP primarily is synthesized and secreted in response to myocyte stretch. Another source of proBNP can be the intima of coronary arteries (30). Therefore, the possible mechanism behind the increase of NTproBNP in KD patients with coronary aneurysm could be explained by the micro-damage of intima of dilated coronary arteries by turbulent bloodstream that results in releasing proBNP (31). Also, two other mechanisms has been postulated including local myocardial inflammation with subsequent production of cytokines, stimulate BNP secretion and local areas of ischemia which can affect the pericardium, myocardium, endocardium, and coronary arteries during the acute phase of $\operatorname{KD}(32,33)$.

Previously the optimum cut-off points for NT-proBNP levels in for diagnosing the coronary artery aneurysm in KD patients were indicated to be at $1000 \mathrm{pg} / \mathrm{ml}$ with a sensitivity of $83 \%$ and a specificity of $68 \%$ by Kaneko et al. and to be at $1300 \mathrm{pg} / \mathrm{ml}$ with $95 \%$ sensitivity and $85 \%$ specificity by Yoshimura et al. which were both close to the occupied cut-off point in our study (34). However, a very recent study of Jung et al. found a lower cut-off point of $515.4 \mathrm{pg} / \mathrm{ml}$ with a sensitivity of $78.26 \%$ and a specificity of $61.63 \%$ (22). The high values of sensitivity and specificity of NT-proBNP at the cut-off point of 1354 $\mathrm{pg} / \mathrm{ml}$ in our study could be due to the evaluation of perivascular brightness of coronary arteries as the preceding factor of developing of coronary artery aneurysm. Also, due to a relatively small number of included patients in the study, we may have missed some overlapping patients. Therefore, we should note some limitations of our study. As far as the appropriate and accessible setting for our study was only the children teaching hospital center, only a limited number of patients with KD, even after extending the study duration, were admitted to the study setting. Also, due to the paucity of patients with cardiac involvement, we could not properly evaluate the association between NT-proBNP levels and severity of cardiac involvement. Since that numerous studies have failed to establish the applicability of routine serum tests (e.g. weight blood cells count, c-reactive protein, erythrocyte sedimentation rate) for diagnosing the cardiac involvement in KD patients, we did not evaluate those serum tests.

\section{Conclusion}

NT-proBNP is elevated in KD patients particularly in those with cardiac involvement; however, cTnl does not change in the serum of KD patients. NT-proBNP can detect the presence of cardiac involvement with an excellent power and high rates of sensitivity and specificity. Therefore, in KD patients with high levels of NT-proBNP, complete evaluation, more rigorous therapy, and closer follow-ups should be considered.

\section{Abbreviations}

Kawasaki Disease (KD) 
Intravenous immunoglobulin (IVIG),

Serum N-terminal pro-brain natriuretic peptide (NT-proBNP)

Transthoracic echocardiography (TTE)

Cardiac troponin I (cTnl)

\section{Declarations}

Ethics approval and consent to participate: Ethical clearance was sought from medical ethics committee of Tabriz University of Medical Sciences, Tabriz, Iran. Written informed consent was obtained from a parent or guardian of the participants.

Consent for publication: Not applicapble.

Acknowledgements: We would like to thank the members of the pediatrics reasearch center of the Tabriz University of Medical Sciences for their kind help that greatly improved the manuscript. The datasets during and/or analyzed during the current study are available from the corresponding author on reasonable request.

Funding: This study was funded by the Tabriz University of Medical Sciences.

Availability of data and materials: All Data and material collected during this study are available from the corresponding author upon reasonable request.

Authors' contributions: Conceived the idea: AM. Designed the study methodology: AM, MS, SG. Conducted the study: AK, AM, SRSE. Analyzed the data: SG, SRSE. Interpreted the results: MS, SG, AM. Wrote the draft manuscript: SRSE, AK. Revised and edited the final manuscript: AM, SG. Approved the manuscript: AM, AK, MS, SG, SRSE.

Competing interests: The authors declare that they have no competing interests.

\section{References}

1. Lin M-T, Wu M-H. The global epidemiology of Kawasaki disease: Review and future perspectives. Global cardiology science \& practice. 2017;2017(3).

2. Mossberg M, Segelmark M, Kahn R, Englund M, Mohammad AJ. Epidemiology of primary systemic vasculitis in children: a population-based study from southern Sweden. Scandinavian journal of rheumatology. 2018;47(4):295-302.

3. Newburger JW, Takahashi M, Gerber MA, Gewitz MH, Tani LY, Burns JC, et al. Diagnosis, treatment, and long-term management of Kawasaki disease: a statement for health professionals from the 
Committee on Rheumatic Fever, Endocarditis and Kawasaki Disease, Council on Cardiovascular Disease in the Young, American Heart Association. Circulation. 2004;110(17):2747-71.

4. Caguit PI, Tee CA, Dans LF. AB1010 Cardiac Involvement in Kawasaki Disease Patients in Philippine General Hospital: A Retrospective Study2015. 1236.3- $p$.

5. Yutani C, Okano K, Kamiya T, Oguchi K, Kozuka T, Ota M, et al. Histopathological study on right endomyocardial biopsy of Kawasaki disease. Heart. 1980;43(5):589-92.

6. Yonesaka S, Takahashi T, Matubara T, Nakada T, Furukawa H, Tomimoto K, et al. Histopathological study on Kawasaki disease with special reference to the relation between the myocardial sequelae and regional wall motion abnormalities of the left ventricle. Japanese circulation journal. 1992;56(4):352-8.

7. Arnold R, Goebel B, Ulmer HE, Gorenflo M, Poerner TC. An exercise tissue Doppler and strain rate imaging study of diastolic myocardial dysfunction after Kawasaki syndrome in childhood. Cardiology in the Young. 2007;17(5):478-86.

8. Tierney ESS, Newburger JW, Graham D, Baker A, Fulton DR, Colan SD. Diastolic function in children with Kawasaki disease. International journal of cardiology. 2011;148(3):309-12.

9. R. Kliegman, B. Stanton, J. St. Geme, Schor N. Nelson textbook of pediatrics 21 th ed. United States of America: Hal B Jenson. 2019.

10. Rawat A, Singh S. Biomarkers for diagnosis of Kawasaki disease. Indian pediatrics. 2015;52(6):4734.

11. Parthasarathy P, Agarwal A, Chawla K, Tofighi T, Mondal TK. Upcoming biomarkers for the diagnosis of Kawasaki disease: a review. Clinical biochemistry. 2015;48(16-17):1188-94.

12. Wu L, Chen Y, Zhong S, Li Y, Dai X, Di Y. Blood N-terminal pro-brain natriuretic peptide and interleukin17 for distinguishing incomplete Kawasaki disease from infectious diseases. Indian pediatrics. 2015;52(6):477-80.

13. Nakamura Y, Yanagawa H, Ojima T, Kawasaki T, Kato H. Cardiac sequelae of Kawasaki disease among recurrent cases. 1998;78(2):163-5.

14. Januzzi Jr JL, Filippatos G, Nieminen M, Gheorghiade M. Troponin elevation in patients with heart failure: on behalf of the third Universal Definition of Myocardial Infarction Global Task Force: Heart Failure Section. European heart journal. 2012;33(18):2265-71.

15. Talwar S, Squire IB, Downie PF, Davies JE, Ng LL. Plasma N terminal pro-brain natriuretic peptide and cardiotrophin 1 are raised in unstable angina. Heart (British Cardiac Society). 2000;84(4):421-4.

16. Tromp J, Khan MAF, Klip IT, Meyer S, de Boer RA, Jaarsma T, et al. Biomarker Profiles in Heart Failure Patients With Preserved and Reduced Ejection Fraction. Journal of the American Heart Association. 2017;6(4):e003989.

17. Gehle P, Robinson PN, Heinzel F, Edelmann F, Yigitbasi M, Berger F, et al. NT-proBNP and diastolic left ventricular function in patients with Marfan syndrome. International journal of cardiology Heart \& vasculature. 2016;12:15-20. 
18. Kim M, Kim K. Elevation of cardiac troponin I in the acute stage of Kawasaki disease. Pediatric cardiology. 1999;20(3):184-8.

19. Checchia PA, Borensztajn J, Shulman ST. Circulating cardiac troponin I levels in Kawasaki disease. Pediatric cardiology. 2001;22(2):102-6.

20. Kaneko K, Yoshimura K, Ohashi A, Kimata T, Shimo T, Tsuji SJPC. Prediction of the Risk of Coronary Arterial Lesions in Kawasaki Disease by Brain Natriuretic Peptide. 2011;32(8):1106-9.

21. Iwashima S, Ishikawa TJWJoP. B-type natriuretic peptide and N-terminal pro-BNP in the acute phase of Kawasaki disease. 2013;9(3):239-44.

22. Jung JY, Ham EM, Kwon H, Kwak YH, Kim DK, Lee JH, et al. N-terminal pro-brain natriuretic peptide and prediction of coronary artery dilatation in hyperacute phase of Kawasaki disease. The American Journal of Emergency Medicine. 2019;37(3):468-71.

23. Miura M, Kobayashi T, Kaneko T, Ayusawa M, Fukazawa R, Fukushima N, et al. Association of Severity of Coronary Artery Aneurysms in Patients With Kawasaki Disease and Risk of Later Coronary Events. JAMA pediatrics. 2018;172(5):e180030-e.

24. Yim D, Burgner D, Cheung M. Echocardiography in Kawasaki disease. Echocardiography-in specific diseases: IntechOpen; 2012.

25. Manlhiot C, Millar K, Golding F, McCrindle BW. Improved classification of coronary artery abnormalities based only on coronary artery z-scores after Kawasaki disease. Pediatric cardiology. 2010;31(2):242-9.

26. Miura M, Kobayashi T, Kaneko T, Ayusawa M, Fukazawa R, Fukushima N, et al. Association of Severity of Coronary Artery Aneurysms in Patients With Kawasaki Disease and Risk of Later Coronary EventsAssociation Between Coronary Artery Aneurysm Severity in Kawasaki Disease and Later Coronary EventsAssociation Between Coronary Artery Aneurysm Severity in Kawasaki Disease and Later Coronary Events. JAMA Pediatrics. 2018;172(5):e180030-e.

27. Takahashi M, Mason WH, Acherman RJ, Lewis AB, Szmuszkovicz JR, Wong PC, et al. Is perivascular echo brightness a reliable marker of coronary arteritis in acute Kawasaki syndrome? Pediatric Research. 2003;53(1):177.

28. Freeman AF, Shulman ST. Issues in the diagnosis of Kawasaki disease. Progress in Pediatric cardiology. 2004;19(2):123-8.

29. Lin YJ, Lin IC, Yu HR, Kuo HC, Yang KD, Chang WC, et al. Tricuspid regurgitation in acute phase of Kawasaki disease associated with intensive care unit admission. Pediatric cardiology. 2013;34(2):250-5.

30. Chung CP, Solus JF, Oeser A, Avalos I, Kurnik D, Raggi P, et al. N-terminal pro-brain natriuretic peptide in systemic lupus erythematosus: relationship with inflammation, augmentation index, and coronary calcification. The Journal of rheumatology. 2008;35(7):1314-9.

31. Abou Sherif S, Ozden Tok O, Taşköylü Ö, Goktekin O, Kilic ID. Coronary Artery Aneurysms: A Review of the Epidemiology, Pathophysiology, Diagnosis, and Treatment. Front Cardiovasc Med. 2017;4:24-. 
32. Kawamura T, Wago M, Kawaguchi H, Yuge MT, Masako. Plasma brain natriuretic peptide concentrations in patients with Kawasaki disease. Pediatrics International. 2000;42(3):241-8.

33. Sato N, Sagawa K, Sasaguri Y, Inoue O, Kato H. Immunopathology and cytokine detection in the skin lesions of patients with kawasaki disease. The Journal of Pediatrics. 1993;122(2):198-203.

34. Yoshimura K, Kimata T, Mine K, Uchiyama T, Tsuji S, Kaneko K. N-Terminal Pro-Brain Natriuretic Peptide and Risk of Coronary Artery Lesions and Resistance to Intravenous Immunoglobulin in Kawasaki Disease. The Journal of Pediatrics. 2013;162(6):1205-9.

\section{Table}

Table 1. Characteristics of KD patients with or without cardiac involvement

\begin{tabular}{lllll} 
& & $\begin{array}{l}\text { Cardiac } \\
\text { involvement }\end{array}$ & $\begin{array}{l}\text { Normal } \\
\text { echocardiography }\end{array}$ & $\begin{array}{l}\text { p- } \\
\text { value }\end{array}$ \\
\hline Age & $2(2)$ & $3(2)$ & 0.343 \\
\hline Sex & Male & $4(100)$ & $21(72.4)$ & 0.001 \\
\cline { 2 - 5 } & Female & 0 & $8(27.6)$ & \\
\hline $\begin{array}{l}\text { Time duration from onset of KD till referring to } \\
\text { hospital (day) }\end{array}$ & $8.25 \pm 1.71$ & $7.75 \pm 2.22$ & 0.491 \\
\hline cTnl & Less than 0.35 & Less than 0.35 & 1.000 \\
\hline NT-proBNP & $3716.3(2114.3)$ & $352.8(352.8)$ & 0.001
\end{tabular}

\section{Figures}




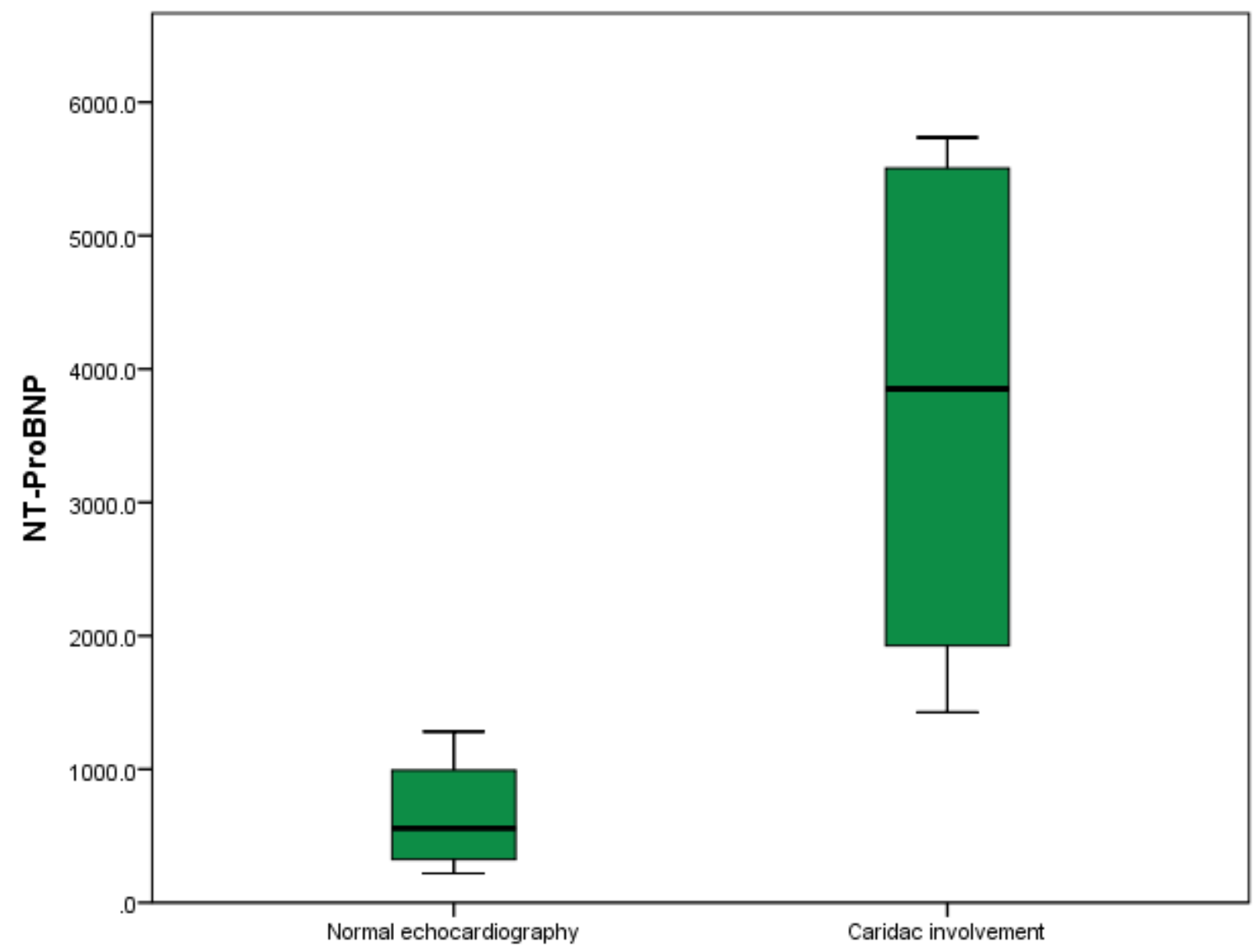

Figure 1

Boxplots of NT-proBNP levels in KD patients based on cardiac involvement 


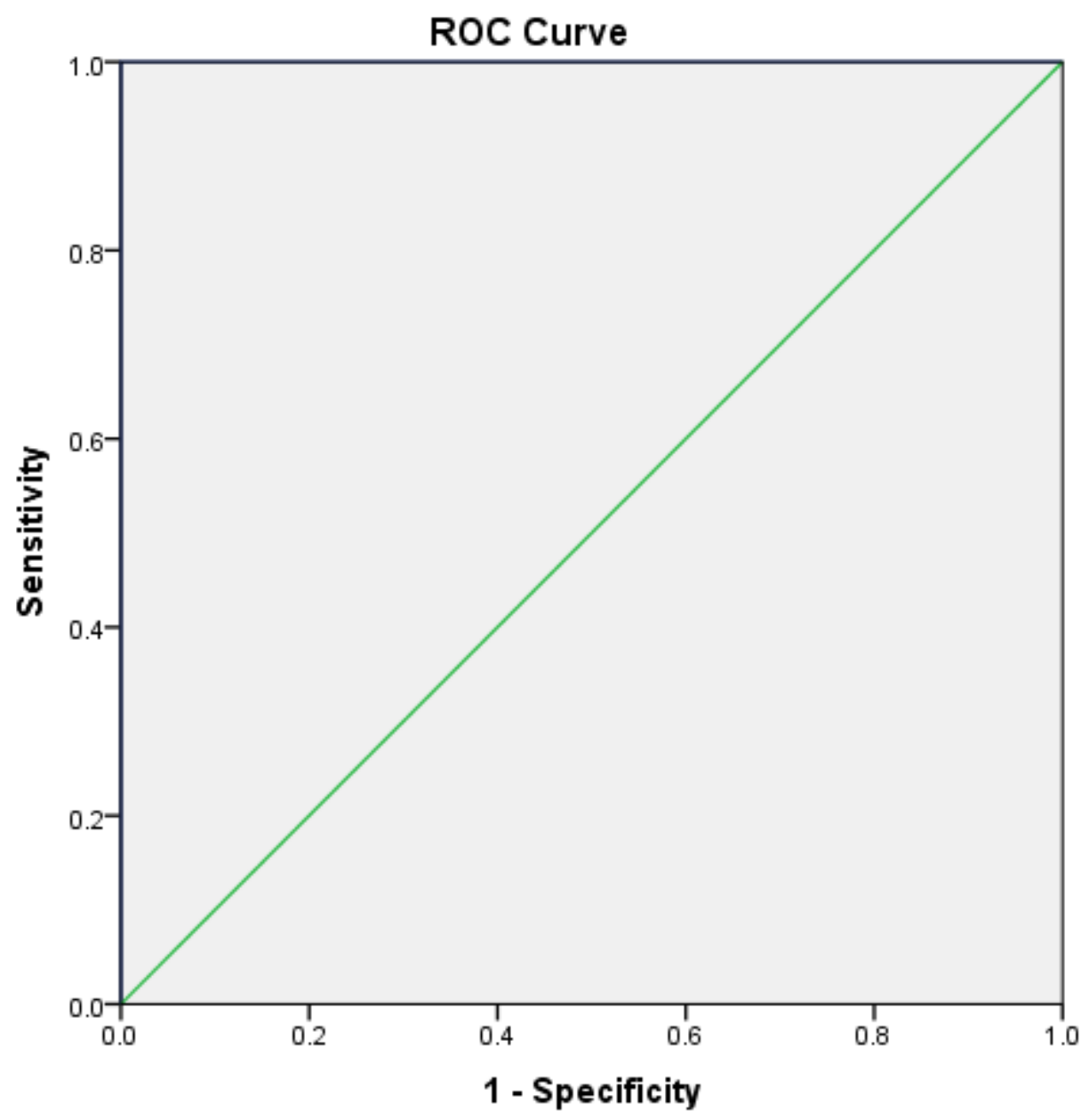

Figure 2

ROC analysis for power of the NT-proBNP in predicting the cardiac involvement in KD patients

\section{Supplementary Files}

This is a list of supplementary files associated with this preprint. Click to download.

- STROBEchecklistv4combined.pdf 